\title{
Spaces of polynomials with roots of bounded multiplicity
}

\author{
by
}

\author{
M. A. Guest (Rochester, NY, and Tokyo), A. Kozlowski (Toyama) \\ and K. Y a mag u c hi (Tokyo)
}

\begin{abstract}
We describe an alternative approach to some results of Vassiliev ([Va1]) on spaces of polynomials, by applying the "scanning method" used by Segal ([Se2]) in his investigation of spaces of rational functions. We explain how these two approaches are related by the Smale-Hirsch Principle or the h-Principle of Gromov. We obtain several generalizations, which may be of interest in their own right.
\end{abstract}

\section{Introduction}

Polynomials and rational functions. The principal motivation for this paper derives from work of Vassiliev ([Va1], [Va2]), in which he describes a general method for calculating the cohomology of certain spaces of polynomial functions (and more generally, "complements of discriminants"). As his paradigmatic example, he takes the space $\mathrm{P}_{n}^{d}(\mathbb{R})$ of real polynomials of the form

$$
x^{d}+a_{d-1} x^{d-1}+\ldots+a_{0}
$$

which have no $n$-fold real roots (but may have complex ones of arbitrary multiplicity!) There is a "jet map" $\mathrm{P}_{n}^{d}(\mathbb{R}) \rightarrow \Omega \mathbb{R} P^{n-1}$ given by $f \mapsto\left[f ; f^{\prime} ; \ldots\right.$ $\left.\ldots ; f^{(n-1)}\right]$, and the image of this map lies in a component $\Omega_{[d]} \mathbb{R} P^{n-1}$, where $[d]=d \bmod 2$. One then has:

Theorem (Vassiliev). If $n \geq 4$, the jet $\operatorname{map} \mathrm{P}_{n}^{d}(\mathbb{R}) \rightarrow \Omega_{[d]} \mathbb{R} P^{n-1}$ is a homotopy equivalence up to dimension $([d / n]+1)(n-2)-1$.

To say that a continuous map $f: X \rightarrow Y$ is a homotopy equivalence up to dimension $d$ means that the induced homomorphism $f_{*}: \pi_{j}(X) \rightarrow \pi_{j}(Y)$ of homotopy groups is bijective when $j<d$ and surjective when $j=d$.

It follows from the theorem that the $d(n-2)$-skeleton of the space $\mathrm{P}_{n}^{n d}(\mathbb{R})$ realizes the first $d+1$ cells in the well known cell decomposition $\Omega_{[d]} \mathbb{R} P^{n-1} \simeq$ $\Omega S^{n-1} \simeq e^{0} \cup e^{n-2} \cup e^{2(n-2)} \cup \ldots$ of the based loop space of the sphere.

1991 Mathematics Subject Classification: 55P35, 58D15, 57R45. 
Homological considerations show that $\mathrm{P}_{n}^{n d}(\mathbb{R})$ has no homology above dimension $d(n-2)$, so in fact $\mathrm{P}_{n}^{n d}(\mathbb{R})$ realizes these first $d+1$ cells exactly.

Vassiliev also considers the space $\mathrm{SP}_{n}^{d}(\mathbb{C})$ of complex polynomials without $n$-fold complex roots, but states an analogue of the above theorem only for $n=2$ (and for homology groups), which gives a well known theorem of May and Segal (see [Se1]) on the configuration space of distinct points in $\mathbb{C}$. For arbitrary $n \geq 2$ one has the following theorem, whose proof we shall give later on:

Theorem (Theorems 2.4 and 2.9). The jet map

$$
\operatorname{SP}_{n}^{d}(\mathbb{C}) \rightarrow \Omega_{d}^{2} \mathbb{C} P^{n-1}, \quad f \mapsto\left[f ; f^{\prime} ; \ldots ; f^{(n-1)}\right],
$$

is a homotopy equivalence up to dimension $(2 n-3)[d / n]$ if $n \geq 3$, and a homology equivalence up to dimension $(2 n-3)[d / n]$ if $n=2$.

In particular, for $n \geq 3$, we have a homotopy equivalence

$$
\lim _{d \rightarrow \infty} \mathrm{SP}_{n}^{d}(\mathbb{C}) \rightarrow \Omega_{0}^{2} \mathbb{C} P^{n-1} .
$$

Remark. We deliberately write $\mathrm{SP}_{n}^{d}(\mathbb{C})$-rather than $\mathrm{P}_{n}^{d}(\mathbb{C})$-in the complex case, because $\mathrm{SP}_{n}^{d}(\mathbb{C})$ is a subspace of the symmetric product $\mathrm{SP}^{d}(\mathbb{C})$ (the space of all complex polynomials of degree $d$ ). Note that $\mathrm{P}_{n}^{d}(\mathbb{R})$ is not a subspace of $\mathrm{SP}^{d}(\mathbb{R})$.

To summarize, we may say that the space of real polynomials without $n$-fold real roots is a model for $\Omega \mathbb{R} P^{n-1}$, and the space of complex polynomials without $n$-fold complex roots is a model for $\Omega^{2} \mathbb{C} P^{n-1}$. We shall show later that the space of complex polynomials without $n$-fold real roots is a model for (the universal covering of) $\Omega \mathbb{C} P^{n-1}$, and that the space of real polynomials without $n$-fold complex roots is a model for the subspace of $\Omega^{2} \mathbb{C} P^{n-1}$ consisting of maps $f: \mathbb{C} \cup\{\infty\} \rightarrow \mathbb{C} P^{n-1}$ such that $f(\bar{z})=\overline{f(z)}$.

Let $\operatorname{Hol}_{d}\left(S^{2}, \mathbb{C} P^{n-1}\right)$ denote the space consisting of all holomorphic (i.e. algebraic) maps $h: S^{2} \rightarrow \mathbb{C} P^{n-1}$ of degree $d$ which satisfy $h(\infty)=$ $[1 ; 0 ; \ldots ; 0]$. Concerning this space, one has the following theorem of [Se2]:

TheORem (Segal). For $n \geq 2$ the inclusion $\operatorname{Hol}_{d}\left(S^{2}, \mathbb{C} P^{n-1}\right) \rightarrow \Omega_{d}^{2} \mathbb{C} P^{n-1}$ is a homotopy equivalence up to dimension $d(2 n-3)$.

This theorem implies that

$$
\lim _{d \rightarrow \infty} \operatorname{Hol}_{d}\left(S^{2}, \mathbb{C} P^{n-1}\right) \simeq \Omega_{0}^{2} \mathbb{C} P^{n-1} .
$$

Combining (1) and (2), we obtain (for $n \geq 3$ ) the existence of a homotopy equivalence

$$
\lim _{d \rightarrow \infty} \operatorname{SP}_{n}^{d}(\mathbb{C}) \simeq \lim _{d \rightarrow \infty} \operatorname{Hol}_{d}\left(S^{2}, \mathbb{C} P^{n-1}\right)
$$


It turns out that there is an explicit description of this homotopy equivalence:

Proposition (Corollary 2.8). The jet map

$$
\mathrm{SP}_{n}^{d}(\mathbb{C}) \rightarrow \operatorname{Hol}_{d}\left(S^{2}, \mathbb{C} P^{n-1}\right), \quad f \mapsto\left[f ; f^{\prime} ; \ldots ; f^{(n-1)}\right],
$$

induces the homotopy equivalence (3).

It was proved by Vassiliev ([Va1], [Va2]) that, if $n \geq 2$, there is a stable homotopy equivalence between $\mathrm{SP}_{n}^{n d}(\mathbb{C})$ and $\operatorname{Hol}_{d}\left(S^{2}, \mathbb{C} P^{n-1}\right)$. The case $n=$ 2 of this result was first proved in [CCMM]. In [GKY2] (and independently in [Kl2]) it was shown that these spaces are in fact homotopy equivalent when $n \geq 3$.

Broadly speaking, two methods of proving theorems of the above types appear in the literature. One may be described as "comparison of spectral sequences", after defining a suitable filtration of each of the spaces concerned. This was introduced in [Va1], [Va2] and independently in [CCMM]. The other uses a "scanning construction"; this method, due to Segal, was developed in [Mc1], [Mc2], [Se2], [Bo], [GKY1], and [Gu1]. We shall use the scanning method here to give proofs of the above results (including Vassiliev's original theorem). We shall also prove various further generalizations, which we describe next.

Equivariant homotopy equivalences. The spaces $\mathrm{SP}_{n}^{d}(\mathbb{C}), \mathrm{P}_{n}^{d}(\mathbb{R})$ are examples of a more general construction. Let $X, Y$ be subspaces of $\mathbb{C}$, and let $\mathrm{P}_{Y, n}^{d}(X)$ denote the space of complex monic polynomials $f$ of degree $d$ such that (i) $f(X) \subseteq X$ and (ii) $f$ has no $n$-fold roots in $Y$. Thus, $\mathrm{P}_{\mathbb{C}, n}^{d}(\mathbb{C})=\mathrm{SP}_{n}^{d}(\mathbb{C})$ and $\mathrm{P}_{\mathbb{R}, n}^{d}(\mathbb{R})=\mathrm{P}_{n}^{d}(\mathbb{R})$, and we have seen that these spaces provide finite-dimensional models for $\Omega^{2} \mathbb{C} P^{n-1}$ and $\Omega \mathbb{R} P^{n-1}$ respectively.

In a similar way, we shall see that the spaces $\mathrm{P}_{\mathbb{C}, n}^{d}(\mathbb{R})$ and $\mathrm{P}_{\mathbb{R}, n}^{d}(\mathbb{C})$ provide models for loop spaces (as mentioned above). Now, $\mathbb{R}$ is the fixed point set of the involution $\theta: \mathbb{C} \rightarrow \mathbb{C}, \theta(z)=\bar{z}$, and this involution extends naturally to $\mathrm{P}_{\mathbb{C}, n}^{d}(\mathbb{C})$ and $\mathrm{P}_{\mathbb{R}, n}^{d}(\mathbb{C})$, and to the corresponding loop spaces. Using $\theta$, the four results concerning $\mathrm{P}_{Y, n}^{d}(X)$ (with $X, Y=\mathbb{R}$ or $\mathbb{C}$ ) can be summarized as follows:

TheOREM (Theorem 3.7). There is a $\theta$-equivariant homotopy equivalence

$$
\lim _{d \rightarrow \infty} \mathrm{P}_{Y, n}^{d}(\mathbb{C}) \rightarrow \operatorname{Map}\left(Y^{+}, S^{2 n-1}\right)
$$

(if $n \geq 4$ ), where $Y=\mathbb{R}$ or $\mathbb{C}, Y^{+}=\mathbb{R} \cup\{\infty\}$ or $\mathbb{C} \cup\{\infty\}$, and Map indicates the space of basepoint preserving continuous maps.

By [JS], a $G$-map $\phi: A \rightarrow B$ of $G$-spaces is an equivariant homotopy equivalence if and only if the maps $\phi^{H}: A^{H} \rightarrow B^{H}$ of fixed point sets are 
homotopy equivalences for all subgroups $H$ of $G$. In the above theorem, $G$ is a group with two elements, so it has precisely two subgroups.

In [Se2], Segal pointed out that the map $\lim _{d \rightarrow \infty} \operatorname{Hol}_{d}\left(S^{2}, \mathbb{C} P^{n-1}\right) \rightarrow$ $\Omega_{0}^{2} \mathbb{C} P^{n-1}$ is an equivariant homotopy equivalence in the same way; this is equivalent to two statements, one for complex rational functions and one for real rational functions. In this case also we have two additional "mixed" spaces (which were not discussed by Segal).

Holomorphic maps. Another generalization of Vassiliev's theorem is obtained by imposing "conditions of bounded multiplicity" on the polynomials appearing in Segal's theorem. If we change the basepoint condition for $f \in \operatorname{Hol}_{d}\left(S^{2}, \mathbb{C} P^{n-1}\right)$ to $f(\infty)=[1 ; \ldots ; 1]$, then $f$ corresponds to an $n$-tuple $\left(p_{1}, \ldots, p_{n}\right)$ of complex monic polynomials of degree $d$ such that $p_{1}, \ldots, p_{n}$ have no common root. For $m \geq 2$, we may define a subspace $\operatorname{Hol}_{d}^{m}\left(S^{2}, \mathbb{C} P^{n-1}\right)$ of $\operatorname{Hol}_{d}\left(S^{2}, \mathbb{C} P^{n-1}\right)$ by imposing the additional condition that each $p_{i}$ belong to $\mathrm{SP}_{m}^{d}(\mathbb{C})$. The scanning construction then shows that $\operatorname{Hol}_{d}^{m}\left(S^{2}, \mathbb{C} P^{n-1}\right)$ is a model for the double loop space $\Omega^{2} A_{n, m}$, where

$$
A_{n, m}=\left\{\left(v_{1}, \ldots, v_{n}\right) \in\left(\mathbb{C}^{m_{-}}\{0\}\right)^{n} \mid\left(\left(v_{1}\right)_{1}, \ldots,\left(v_{n}\right)_{1}\right) \neq(0, \ldots, 0)\right\} .
$$

The space $A_{n, m}$ is an example of (the complement of) a "subspace arrangement"; the topology of such spaces has been studied intensively (see, for example, part III of [GM], as well as [Va1]).

Segal's method extends to the case of $\operatorname{Hol}\left(S^{2}, X\right)$, where $X$ is a toric variety (see [GKY1], [Gu1], [Gu2]). By imposing conditions of bounded multiplicity on these spaces, we obtain many further results of the above type. In each case, a subspace of $\operatorname{Hol}\left(S^{2}, X\right)$ gives a (finite-dimensional) topological approximation to the double loop space of the complement of a certain subspace arrangement. The "equivariant" results mentioned above also extend to these examples.

The above results may be generalized in a different way by replacing $\mathbb{C}$ by an open Riemann surface $\Sigma$, and $S^{2}\left(=\mathbb{C}^{+}\right)$by the one-point compactification $\Sigma^{+}$. One obtains (for example) an equivalence up to some dimension between $\operatorname{SP}_{n}^{d}(\Sigma)$ and the space $\operatorname{Map}\left(\Sigma^{+}, \mathbb{C} P^{n-1}\right)$ of based maps. (Here, $\mathrm{SP}_{n}^{d}(\Sigma)$ is interpreted as a subspace of the $d$ th symmetric product $\mathrm{SP}^{d}(\Sigma)$.) For simplicity we shall restrict our exposition in this paper to the case $\Sigma=\mathbb{C}$, referring to [Mc1] for the method of extension to general $\Sigma$ (and to open manifolds $\Sigma$ of arbitrary dimension). However, it should be emphasized that the extension to manifolds other than $\mathbb{C}$ appears to be an advantage of the scanning method.

Finally, we mention that the space $\operatorname{Hol}\left(S^{2}, X\right)$ may be identified settheoretically (although not topologically) with the space " $\operatorname{Hol}\left(S^{1}, X\right)$ " which plays a role in the Gromov-Floer theory of holomorphic curves (see for example $[\mathrm{Fu}])$. It is noted in Section 3 of [CJS] that $\operatorname{Hol}\left(S^{1}, \mathbb{C} P^{n-1}\right)$ is 
homotopy equivalent to the universal covering space of $\Omega \mathbb{C} P^{n-1}$. This fact can be proved by the methods described here, using $n$-tuples $\left(p_{1}, \ldots, p_{n}\right)$ of polynomials which have no common root on $S^{1}$.

The h-Principle. The "scanning method" applies to all these examples because in each case the space of polynomials involved can be identified with a certain space of "labelled configurations". By a labelled configuration we mean a finite set of distinct points (usually in $\mathbb{C}$ ) where each point $z$ is labelled by an element $m$ of a fixed partial monoid $M$. The set of all such labelled configurations is topologized in the usual way, except that two labelled points $\left(z_{1}, m_{1}\right),\left(z_{2}, m_{2}\right)$ are allowed to move towards each other and coalesce $\left({ }^{1}\right)$, producing a new labelled point $\left(z, m_{1}+m_{2}\right)$, if the sum $m_{1}+m_{2}$ exists in $M$. More generally still, Kallel ([Kl1]) has formulated the notion of "particle space".

A deeper explanation for the existence of results of the above type is suggested by Vassiliev, in terms of the Smale-Hirsch Principle. In its most general form, this is also known as the $h$-Principle of Gromov (see [Gr]). The relevant version of this principle says that - under certain conditions - the space of maps $M \rightarrow N$ whose $k$-jets avoid a "discriminant variety" $S$ in the jet space $J^{k}(M, N)$ is homotopy equivalent to the space consisting of all sections of the bundle $J^{k}(M, N)-S \rightarrow M$. We shall describe a precise relation between the scanning method and the h-Principle. The existence of such a relation should not be surprising, as the scanning method was in fact based on earlier ideas of Gromov (see [Mc2]). However, the connection with Gromov's work has been obscured in recent years by an emphasis (in the algebraic topology literature) on configuration spaces, so it seemed worthwhile to explain here the original point of view. Indeed, it might be argued that the h-Principle gives the most natural approach to all "stable" results of the type considered here.

The paper is organized as follows. In $\S 2$ we discuss for simplicity only the fundamental examples $\mathrm{SP}_{n}^{d}(\mathbb{C})$ and $\operatorname{Hol}_{d}\left(S^{2}, \mathbb{C} P^{n-1}\right)$, and the relation between them. Various modifications of these examples (including Vassiliev's original situation) are described in $\S 3$. Finally, in $\S 4$, we present the most general situation, and we explain the relation with the h-Principle.

Acknowledgements. We thank Sadok Kallel for sending us his preprints [Kl1], [Kl2], and Dai Tamaki for informing us about the work [Kt] of Fumiko Kato. Similar results to our Theorem 2.2 were obtained independently both in [Kl2] and in [Kt]. The first author was partially supported by a grant from the US National Science Foundation, and the third author by a grant from the Ministry of Education of Japan.

$\left({ }^{1}\right)$ This is somewhat different to the usual notion of labelled configuration in topology, where $M$ is simply a set. In that case, distinct labelled points are never allowed to coalesce. 


\section{The fundamental example}

Basic definitions. For any space $X$, we denote by $\operatorname{SP}^{d}(X)$ the $d$ th symmetric product of $X$. By definition, this is the quotient space $X^{d} / \Sigma_{d}$, where the symmetric group $\Sigma_{d}$ acts on the $d$-fold product $X^{d}$ in the natural way. An element of $\mathrm{SP}^{d}(X)$ may be identified with a formal linear combination $\alpha=\sum_{i=1}^{k} d_{i} x_{i}$, where $x_{1}, \ldots, x_{k}$ are distinct points of $X$ and $d_{1}, \ldots, d_{k}$ are positive integers such that $\sum_{i=1}^{k} d_{i}=d$. We shall refer to $\alpha$ as a "configuration" of points, the point $x_{i}$ having multiplicity $d_{i}$.

In this section we shall be concerned with a subspace $\mathrm{SP}_{n}^{d}(X)$ of $\mathrm{SP}^{d}(X)$, defined as follows:

Definition 2.1. For $n \geq 2, \operatorname{SP}_{n}^{d}(X)=\left\{\sum_{i=1}^{k} d_{i} x_{i} \in \operatorname{SP}^{d}(X) \mid d_{i}<n\right.$ for all $i\}$.

Thus, $\mathrm{SP}_{n}^{d}(X)$ is obtained by imposing a condition of "bounded multiplicity", namely that all points $x_{i}$ (of any configuration) have multiplicity less than $n$. There is a filtration

$$
\mathrm{C}_{d}(X)=\mathrm{SP}_{2}^{d}(X) \subseteq \mathrm{SP}_{3}^{d}(X) \subseteq \ldots \subseteq \mathrm{SP}_{d+1}^{d}(X)=\mathrm{SP}^{d}(X)
$$

where $\mathrm{C}_{d}(X)$ denotes the space of "configurations of $d$ distinct points" in $X$.

If $A$ is a closed subspace of $X$, we define

$$
\mathrm{SP}_{n}^{d}(X, A)=\left\{\sum_{i=1}^{k} d_{i} x_{i} \in \mathrm{SP}_{n}^{d}(X) \mid d_{i}<n \text { if } x_{i} \in X-A\right\} / \sim
$$

where $\alpha \sim \beta$ if and only if $\alpha \cap(X-A)=\beta \cap(X-A)$. Thus, for $\operatorname{SP}_{n}^{d}(X, A)$, points in $A$ are "ignored". When $A$ is nonempty, there is a natural inclusion map

$$
\mathrm{SP}_{n}^{d}(X, A) \rightarrow \mathrm{SP}_{n}^{d+1}(X, A)
$$

given by "adding a fixed point in $A$ ". We define

$$
\operatorname{SP}_{n}(X, A)=\bigcup_{d \geq 1} \operatorname{SP}_{n}^{d}(X, A)
$$

As a set, $\mathrm{SP}_{n}(X, A)$ is bijectively equivalent to $\bigcup_{d \geq 0} \mathrm{SP}_{n}^{d}(X-A)$ (disjoint union), but these two spaces are not in general homeomorphic. For example, if $\sum_{i=1}^{k} d_{i} x_{i} \in \mathrm{SP}_{n}(X, A)$ with $x_{1}, \ldots, x_{k} \in X-A$, then a point $x_{i}$ can move into $A$ and "disappear". In particular, $\operatorname{SP}_{n}(X, A)$ is connected if $X$ is connected.

We shall usually take $X$ to be an open subset of the complex numbers $\mathbb{C}$. Note that $\mathrm{SP}_{n}^{d}(\mathbb{C})$ can be identified with the space of complex polynomials of degree $d$ which are monic, and all of whose roots have multiplicity less than $n$. (The polynomial $\prod_{i=1}^{k}\left(z-x_{i}\right)^{d_{i}}$ corresponds to $\sum_{i=1}^{k} d_{i} x_{i}$.) In this 
special situation, there is a "stabilization map"

$$
\mathrm{SP}_{n}^{d}(\mathbb{C}) \rightarrow \mathrm{SP}_{n}^{d+1}(\mathbb{C})
$$

which may be defined (up to homotopy) as follows. If $U_{d}=\{z \in \mathbb{C}|| z \mid<d\}$, it is obvious that $\mathrm{SP}_{n}^{d}(\mathbb{C})$ is homeomorphic to $\mathrm{SP}_{n}^{d}\left(U_{d}\right)$. Via this identification, the required map

$$
\mathrm{SP}_{n}^{d}\left(U_{d}\right) \rightarrow \mathrm{SP}_{n}^{d+1}\left(U_{d+1}\right)
$$

is defined by $\sum_{i=1}^{k} d_{i} x_{i} \mapsto x+\sum_{i=1}^{k} d_{i} x_{i}$, where $x$ is a fixed point of $U_{d+1}-U_{d}$.

The scanning construction for configuration spaces. To investigate the space $\lim _{d \rightarrow \infty} \mathrm{SP}_{n}^{d}(\mathbb{C})$, we use the "scanning map"

$$
s_{n}^{d}: \mathrm{SP}_{n}^{d}(\mathbb{C}) \rightarrow \Omega^{2} \mathrm{SP}_{n}(\bar{U}, \partial \bar{U})
$$

where $U=\{z \in \mathbb{C}|| z \mid<1\}$. To define this, we write $U(w)=\{z \in \mathbb{C} \mid$ $|z-w|<\varepsilon\}$, where $\varepsilon>0$ is fixed. Let $\alpha=\sum_{i=1}^{k} d_{i} x_{i} \in \operatorname{SP}_{n}^{d}(\mathbb{C})$. Then the map

$$
s_{n}^{d}(\alpha): \mathbb{C} \cup \infty \rightarrow \operatorname{SP}_{n}(\bar{U}, \partial \bar{U})
$$

is defined by

$$
z \mapsto \alpha \cap \bar{U}(z) \in \mathrm{SP}_{n}(\bar{U}(z), \partial \bar{U}(z)) \cong \mathrm{SP}_{n}(\bar{U}, \partial \bar{U}) .
$$

Note that $s_{n}^{d}(\alpha)$ is a basepoint preserving map: the point $\infty$ is always mapped to the empty configuration in $\operatorname{SP}_{n}(\bar{U}, \partial \bar{U})$.

As $\mathrm{SP}_{n}^{d}(\mathbb{C})$ is connected, the image of $s_{n}^{d}$ lies in a connected component of $\Omega^{2} \mathrm{SP}_{n}(\bar{U}, \partial \bar{U})$, which we denote by $\Omega_{d}^{2} \mathrm{SP}_{n}(\bar{U}, \partial \bar{U})$. We shall see later $\left(^{2}\right)$ that $\operatorname{SP}_{n}(\bar{U}, \partial \bar{U}) \simeq \mathbb{C} P^{n-1}$; it is then easy to show that $\Omega_{d}^{2} \mathrm{SP}_{n}(\bar{U}, \partial \bar{U})$ is the " $d$ th component" in the usual sense. The main reason for introducing the map $s_{n}^{d}$ is:

Theorem 2.2. For $n \geq 3$,

$$
\lim _{d \rightarrow \infty} s_{n}^{d}: \lim _{d \rightarrow \infty} \mathrm{SP}_{n}^{d}(\mathbb{C}) \rightarrow \lim _{d \rightarrow \infty} \Omega_{d}^{2} \mathrm{SP}_{n}(\bar{U}, \partial \bar{U}) \simeq \Omega_{0}^{2} \mathrm{SP}_{n}(\bar{U}, \partial \bar{U})
$$

is a homotopy equivalence. For $n=2, \lim _{d \rightarrow \infty} s_{n}^{d}$ is a homology equivalence.

Proof. The proof is similar to the argument of $\S 3$ of [Se2]. A detailed exposition, more suited to the purposes of the present article, is given in Proposition 3.1 of [Gu1]. Since this is an important argument, however, which will reappear in $\S 4$ in connection with the h-Principle, we shall sketch the main ideas here (cf. [Mc2]).

$\left({ }^{2}\right)$ This fact, as well as our Theorem 2.2, was noted independently by Kallel ([Kl2]) and Kato $([\mathrm{Kt}])$. 
For real numbers $x, y \geq 0$, let $D_{[x, y]}=\{z \in \mathbb{C}|x \leq| z \mid \leq y\}$. Consider the commutative diagram

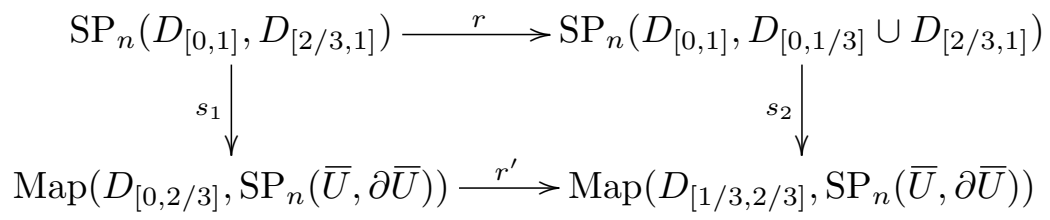

in which the maps $r, r^{\prime}$ are the natural "restriction" maps, and the maps $s_{1}, s_{2}$ are given by scanning. (Here, Map indicates continuous maps.) The map of the theorem is closely related to the map of fibres of the horizontal maps. To obtain the map of the theorem one must modify the method as explained in [Se2], but we ignore these modifications here as our purpose is merely to explain the main points of the argument. After this modification, the map $r$ becomes a quasifibration. It is an elementary fact that the map $r^{\prime}$ is a fibration. The theorem will follow if we prove that the maps $s_{1}, s_{2}$ of total spaces and of base spaces are homotopy equivalences.

In the case of $s_{1}$, this is trivially so. To deal with $s_{2}$, we shall need some new notation. Let $R_{x}=\{z \in \mathbb{C} \mid \operatorname{Re} z \geq x\}$ and $L_{x}=\{z \in \mathbb{C} \mid \operatorname{Re} z \leq x\}$. Then consider the commutative diagrams

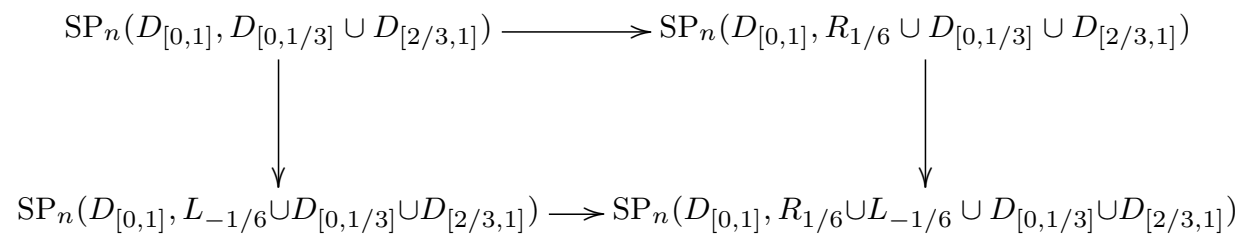

and

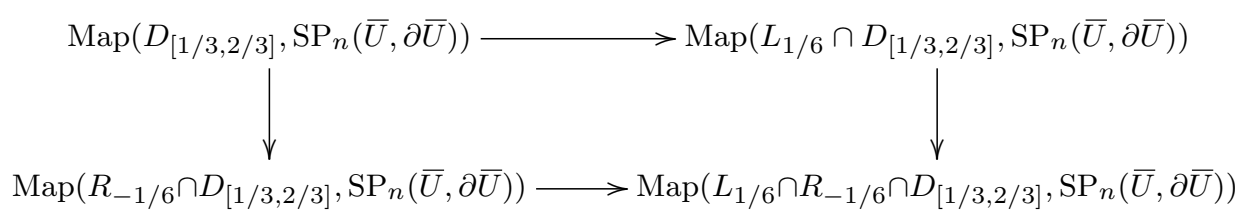

Both diagrams are homotopy Cartesian. There are compatible maps from the first diagram to the second, given by scanning, one of which is the map $s_{2}$. The remaining three maps are homotopy equivalences (because $s_{1}$ is). Hence $s_{2}$ must be a homotopy equivalence also, as required.

The scanning construction for algebraic maps. It is illuminating to convert Theorem 2.2 to a result about polynomial functions with singularities of a certain type. This gives the connection with the work of Vassiliev ([Va1], [Va2]) mentioned in the introduction. We need the following definition: 
Definition 2.3. (1) For $n \geq 2, \mathcal{S P}_{n}^{d}(\mathbb{C})$ denotes the space of (not necessarily monic) complex polynomial functions $f(z)=\sum a_{i} z^{i}$ of degree exactly $d$ such that every root of $f$ has multiplicity less than $n$.

(2) For $n \geq 2$, and any nonempty open subset $X \subseteq \mathbb{C}, \mathcal{S P}_{n}(X)$ denotes the space of complex polynomial functions $f(z)=\sum a_{i} z^{i}$ such that every root of $f$ in $X$ has multiplicity less than $n$, and such that $f$ is not identically zero.

Both $\mathcal{S P}_{n}^{d}(\mathbb{C})$ and $\mathcal{S P}_{n}(X)$ are topologized as subspaces of the space of all complex polynomials. Note that $\mathcal{S P}_{n}(\mathbb{C})$ is bijectively equivalent to the disjoint union $\bigcup_{d>0} \mathcal{S} \mathcal{P}_{n}^{d}(\mathbb{C})$, but these spaces are not homeomorphic because $\mathcal{S P}_{n}(\mathbb{C})$ is connected-roots of polynomials in $\mathcal{S P}_{n}(\mathbb{C})$ are allowed to move to infinity and "disappear".

There is a version of the scanning map for $\mathcal{S P}_{n}^{d}(\mathbb{C})$, namely

$$
\operatorname{scan}: \mathcal{S} \mathcal{P}_{n}^{d}(\mathbb{C}) \rightarrow \operatorname{Map}\left(\mathbb{C}, \mathcal{S P} \mathcal{P}_{n}(U)\right), \quad f \mapsto\left(\left.z \mapsto f\right|_{U(z)}\right),
$$

(where, as in the definition of the earlier scanning map, we use the canonical identification $U(z) \cong U)$. Here, $\operatorname{Map}(A, B)$ denotes the space of continuous maps from $A$ to $B$. The definition of $\mathcal{S P}_{n}^{d}(\mathbb{C})$ suggests that we consider as well the jet map:

$$
\text { jet }: \mathcal{S} \mathcal{P}_{n}^{d}(\mathbb{C}) \rightarrow \operatorname{Map}\left(\mathbb{C}, \mathbb{C}^{n}-\{0\}\right), \quad f \mapsto\left(f, f^{\prime}, \ldots, f^{(n-1)}\right) .
$$

We shall describe a relation between the scanning map for configurations and these two natural maps.

The maps

$$
p: \mathcal{S P}_{n}^{d}(\mathbb{C}) \rightarrow \mathrm{SP}_{n}^{d}(\mathbb{C}), \quad q: \mathcal{S P}_{n}(U) \rightarrow \mathrm{SP}_{n}(\bar{U}, \partial \bar{U})
$$

given by assigning to a polynomial function its roots are the key to this relation. It is obvious that $p$ is a principal fibre bundle, with fibre $\mathbb{C}^{*}$. Moreover, this is a trivial bundle because there is a section, defined by assigning to a point of $\mathrm{SP}_{n}^{d}(\mathbb{C})$ the corresponding monic polynomial. Similarly, the "fibre" of the map $q$ is the space of all polynomials in $\mathcal{S P}_{n}(U)$ whose roots lie outside $U$, and this is homotopy equivalent to $\mathbb{C}^{*}$. We claim that $q$ is in fact a quasifibration. This may be proved using the well known criterion of Dold and Thom, as in the proof of a similar assertion in Lemma 3.3 of [Se2]. Namely, we filter the base space $\operatorname{SP}_{n}(\bar{U}, \partial \bar{U})$ by the number of points in $U$, and use the fact that $q$ is a (trivial) fibre bundle over each successive difference in this filtration. The Dold-Thom "attaching map" has the effect of multiplying polynomials with no roots in $U$ by a fixed polynomial $z-\alpha$, where $\alpha$ lies outside $U$. Since $\alpha$ may be moved continuously to 1 , the corresponding map of $\mathbb{C}^{*}$ is a homotopy equivalence, as required. 
The scanning maps for $\mathrm{SP}_{n}^{d}(\mathbb{C})$ and $\mathcal{S P}_{n}^{d}(\mathbb{C})$ are related by the following commutative diagram. (Diagrams of this type will play a central role in this paper.)

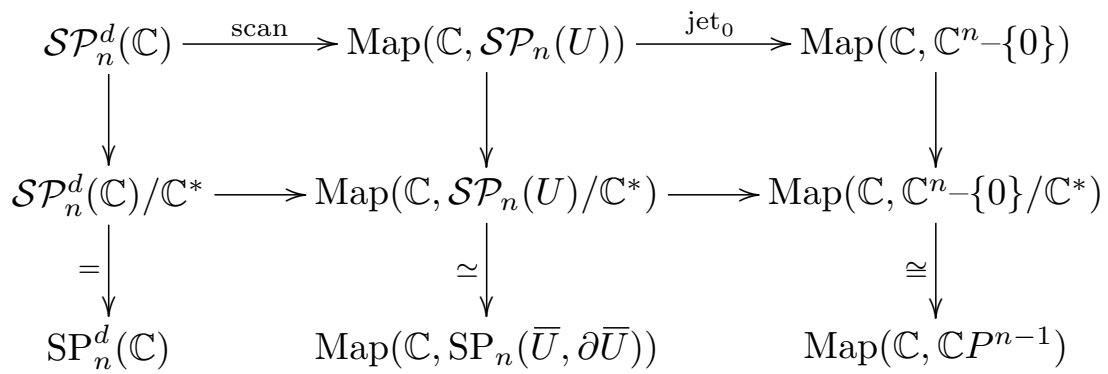

The first and second columns are induced by $p$ and $q$ respectively. The map jet $_{0}$ is induced by the map $f \mapsto\left(f(0), f^{\prime}(0), \ldots, f^{(n-1)}(0)\right)$. Note that the first row of the diagram is simply a factorization of the jet map $\mathcal{S P}_{n}^{d}(\mathbb{C}) \rightarrow$ $\operatorname{Map}\left(\mathbb{C}, \mathbb{C}^{n}-\{0\}\right)$.

Taking into account the behaviour of the scanning map at $\infty$, we see that the second row of this diagram gives a map into $\Omega_{d}^{2} \mathbb{C} P^{n-1}$, which we denote by

$$
j_{n}^{d}: \mathrm{SP}_{n}^{d}(\mathbb{C}) \rightarrow \Omega_{d}^{2} \mathbb{C} P^{n-1} .
$$

With all the necessary preparations behind us, we can now prove our first main result concerning this map:

Theorem 2.4. For $n \geq 3$,

$$
\lim _{d \rightarrow \infty} j_{n}^{d}: \lim _{d \rightarrow \infty} \mathrm{SP}_{n}^{d}(\mathbb{C}) \rightarrow \lim _{d \rightarrow \infty} \Omega_{d}^{2} \mathbb{C} P^{n-1} \simeq \Omega_{0}^{2} \mathbb{C} P^{n-1}
$$

is a homotopy equivalence. For $n=2, \lim _{d \rightarrow \infty} j_{n}^{d}$ is a homology equivalence.

Pr o of. Consider the above commutative diagram, in which $j_{n}^{d}$ is (essentially) the second row. The first part of the second row, i.e. the scanning $\operatorname{map} \mathrm{SP}_{n}^{d}(\mathbb{C}) \rightarrow \operatorname{Map}\left(\mathbb{C}, \mathcal{S P}_{n}(U) / \mathbb{C}^{*}\right)$, gives rise to the map of Theorem 2.2. Hence it is a homotopy equivalence (in the limit $d \rightarrow \infty$ ) if $n \geq 3$, and a homology equivalence if $n=2$.

We claim that the jet map

$$
\text { jet }_{0}: \mathcal{S P}_{n}(U) \rightarrow \mathbb{C}^{n}-\{0\}, \quad f \mapsto\left(f(0), f^{\prime}(0), \ldots, f^{(n-1)}(0)\right),
$$

is a $\mathbb{C}^{*}$-equivariant weak homotopy equivalence. (This implies that the second part of the second row is a weak homotopy equivalence, and hence that the map in the statement of the theorem is a weak homotopy equivalence. But each space in this statement has the homotopy type of a CW-complex, so the map is actually a homotopy equivalence, and the proof of the theorem is complete.) To prove the claim, we use the same direct argument as for 
Proposition 1 of $[\mathrm{Ha}]$ to show that the inclusion

$$
\mathcal{S P}_{n}(U) \rightarrow\left\{f(z)=\sum_{i \geq 0} a_{i} z^{i} \mid\left(f(0), f^{\prime}(0), \ldots, f^{(n-1)}(0)\right) \neq(0, \ldots, 0)\right\}
$$

is a weak homotopy equivalence. On replacing $a_{i}$ by $t a_{i}$ for $i \geq n$, and letting $t \rightarrow 0$, we deduce that $\mathcal{S P}_{n}(U)$ is weakly homotopy equivalent to

$$
\left\{f(z)=\sum_{i=0}^{n-1} a_{i} z^{i} \mid\left(a_{0}, a_{1}, 2 ! a_{2}, \ldots,(n-1) ! a_{n-1}\right) \neq(0, \ldots, 0)\right\} .
$$

The jet map is therefore equivalent to the map

$$
\mathbb{C}^{n_{-}}\{0\} \rightarrow \mathbb{C}^{n_{-}}\{0\}, \quad\left(a_{0}, \ldots, a_{n-1}\right) \mapsto\left(a_{0}, a_{1}, 2 ! a_{2}, \ldots,(n-1) ! a_{n-1}\right),
$$

which is certainly a weak homotopy equivalence. Moreover, all maps here are clearly $\mathbb{C}^{*}$-equivariant.

REMARK. A consequence of (the last part of) this proof is that the space $\mathrm{SP}_{n}(\bar{U}, \partial \bar{U})$, which appears in the scanning construction for configurations, is homotopy equivalent to $\mathbb{C} P^{n-1}$.

Segal's theorem on rational functions. We shall give a brief description of (the stable version of) Segal's theorem ([Se2]) on holomorphic maps, in the spirit of the above discussion.

Definition 2.5. For $n \geq 2$, let $\mathrm{Q}_{d}^{(n-1)}(\mathbb{C})$ be the space of all $n$-tuples $\left(\alpha_{1}, \ldots, \alpha_{n}\right)$ with $\alpha_{i} \in \mathrm{SP}^{d}(\mathbb{C})$ such that $\alpha_{1} \cap \ldots \cap \alpha_{n}=\emptyset$.

Alternatively, $\mathrm{Q}_{d}^{(n-1)}(\mathbb{C})$ is the space of $n$-tuples $\left(p_{1}, \ldots, p_{n}\right)$ of complex polynomials of degree $d$ which are monic and coprime. The polynomials $p_{1}, \ldots, p_{n}$ may be regarded as the homogeneous coordinates of a holomorphic map $F=\left[p_{1} ; \ldots ; p_{n}\right]$ from $\mathbb{C} P^{1}=S^{2}=\mathbb{C} \cup\{\infty\}$ to $\mathbb{C} P^{n-1}$. We have $F(\infty)=[1 ; \ldots ; 1]$ and $[F]=d \in \pi_{2} \mathbb{C} P^{n-1}$. Conversely, it is well known that any holomorphic map $F: \mathbb{C} P^{1} \rightarrow \mathbb{C} P^{n-1}$ satisfying the last two conditions corresponds to an element of $\mathrm{Q}_{d}^{(n-1)}(\mathbb{C})$. This means that $\mathrm{Q}_{d}^{(n-1)}(\mathbb{C})$ may be identified with the space $\operatorname{Hol}_{d}\left(S^{2}, \mathbb{C} P^{n-1}\right)$ of such maps.

There is a natural inclusion map $i_{n}^{d}: \mathrm{Q}_{d}^{(n-1)}(\mathbb{C})=\operatorname{Hol}_{d}\left(S^{2}, \mathbb{C} P^{n-1}\right) \rightarrow$ $\Omega_{d}^{2} \mathbb{C} P^{n-1}$. In [Se2], Segal proves:

Theorem 2.6. For $n \geq 3$,

$$
\lim _{d \rightarrow \infty} i_{n}^{d}: \lim _{d \rightarrow \infty} \mathrm{Q}_{d}^{(n-1)}(\mathbb{C}) \rightarrow \Omega_{0}^{2} \mathbb{C} P^{n-1}
$$

is a homotopy equivalence. For $n=2, \lim _{d \rightarrow \infty} i_{n}^{d}$ is a homology equivalence.

We sketch the proof here, making only a rearrangement of the proof given in [Se2]. 
Definition 2.7. (1) For $n \geq 2, \mathcal{Q}_{d}^{(n-1)}(\mathbb{C})$ denotes the space of $n$-tuples $\left(p_{1}, \ldots, p_{n}\right)$ of (not necessarily monic) complex polynomial functions of degree exactly $d$ such that $p_{1}, \ldots, p_{n}$ have no common root.

(2) For $n \geq 2$, and any nonempty open subset $X \subseteq \mathbb{C}, \mathcal{Q}^{(n-1)}(X)$ denotes the space of $n$-tuples $\left(p_{1}, \ldots, p_{n}\right)$ of complex polynomial functions such that $p_{1}, \ldots, p_{n}$ have no common root in $X$, and such that no $p_{i}$ is identically zero.

There are scanning maps

$$
\mathrm{Q}_{d}^{(n-1)}(\mathbb{C}) \rightarrow \operatorname{Map}\left(\mathbb{C}, \mathrm{Q}^{(n-1)}(\bar{U}, \partial \bar{U})\right)
$$

(in which the definition of $\mathrm{Q}^{(n-1)}(X, Y)$ is analogous to that of $\mathrm{SP}_{n}(X, Y)$ ) and

$$
\mathcal{Q}_{d}^{(n-1)}(\mathbb{C}) \rightarrow \operatorname{Map}\left(\mathbb{C}, \mathcal{Q}^{(n-1)}(U)\right)
$$

The analogue of the jet map in the present situation is the inclusion $\mathcal{Q}_{d}^{(n-1)}(\mathbb{C}) \rightarrow \operatorname{Map}\left(\mathbb{C}, \mathbb{C}^{n}-\{0\}\right)$. The analogue of jet ${ }_{0}$ is simply evaluation at 0 . The analogue of the earlier commutative diagram is:

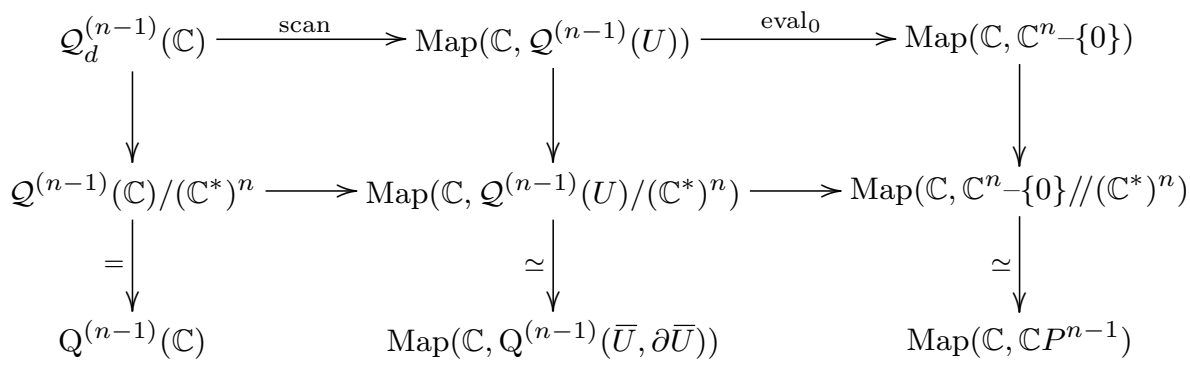

The only new feature here is that $\left(\mathbb{C}^{*}\right)^{n}$ does not act freely on $\mathbb{C}^{n}-\{0\}$; for this reason it is necessary to use the homotopy quotient $\mathbb{C}^{n}-\{0\} / /\left(\mathbb{C}^{*}\right)^{n}$ in order to ensure that each vertical map is a fibration with fibre $\left(\mathbb{C}^{*}\right)^{n}$. (Note however that $\left(\mathbb{C}^{*}\right)^{n}$ acts freely on $\mathcal{Q}^{(n-1)}(U)$, because of the condition that no component of any element of $\mathcal{Q}^{(n-1)}(U)$ is identically zero.)

The proof of Segal's theorem now proceeds as in the case of Theorem 2.4. First, the map under consideration is given by the second row of the diagram (after imposing the basepoint condition at $\infty$ ). Next, the scanning map $\mathrm{Q}_{d}^{(n-1)}(\mathbb{C}) \rightarrow \Omega_{d}^{2} \mathrm{Q}^{(n-1)}(\bar{U}, \partial \bar{U})$ is a homotopy equivalence (in the limit $d \rightarrow \infty$ ), as in Theorem 2.2. And finally, we argue as in the proof of Theorem 2.4 that eval ${ }_{0}$ gives a $\left(\mathbb{C}^{*}\right)^{n}$-equivariant homotopy equivalence $\mathcal{Q}^{(n-1)}(U) \simeq \mathbb{C}^{n}-\{0\}$. (There is an additional complication in the present situation as we have excluded from $\mathcal{Q}^{(n-1)}(U)$ those $\left(p_{1}, \ldots, p_{n}\right)$ which have some component identically zero. However, the removal of this infinitecodimensional space of functions does not affect the homotopy type - see Section 4 of [Se2].) 
REMARK. It follows from the homotopy equivalence $\mathcal{Q}^{(n-1)}(U) \simeq$ $\mathbb{C}^{n}-\{0\}$ that the space $\mathrm{Q}^{(n-1)}(\bar{U}, \partial \bar{U})$ is homotopy equivalent to the homotopy quotient $\mathbb{C}^{n}-\{0\} / /\left(\mathbb{C}^{*}\right)^{n}$. This, in turn, is homotopy equivalent to the "fat wedge" $W_{n}\left(\mathbb{C} P^{\infty}\right)$ of $n$ copies of $\mathbb{C} P^{\infty}$ (see Section 2 of $[\mathrm{Se} 2]$ ).

The first row of the above diagram is simply the natural inclusion map. Thus, we have shown that the natural inclusion $\operatorname{Hol}_{d}\left(S^{2}, \mathbb{C} P^{n-1}\right) \rightarrow$ $\operatorname{Map}_{d}\left(S^{2}, \mathbb{C} P^{n-1}\right)$ may be identified up to homotopy with the scanning map $\mathrm{Q}_{d}^{(n-1)}(\mathbb{C}) \rightarrow \Omega_{d}^{2} \mathrm{Q}^{(n-1)}(\bar{U}, \partial \bar{U})$, which is a homotopy equivalence (in the limit $d \rightarrow \infty$ ). This completes our sketch of the proof of Segal's theorem.

Now we describe the relation between Theorem 2.4 and Segal's theorem. Theorem 2.4 says that a certain map

$$
j_{n}^{d}: \mathrm{SP}_{n}^{d}(\mathbb{C}) \rightarrow \Omega_{d}^{2} \mathbb{C} P^{n-1}
$$

is a homotopy equivalence (when $d \rightarrow \infty$ ). Theorem 2.6 says that a certain map

$$
i_{n}^{d}: \mathrm{Q}_{d}^{(n-1)}(\mathbb{C}) \rightarrow \Omega_{d}^{2} \mathbb{C} P^{n-1}
$$

is a homotopy equivalence (when $d \rightarrow \infty$ ). We have a map

$$
T_{n}^{d}: \mathrm{SP}_{n}^{d}(\mathbb{C}) \rightarrow Q_{d}^{(n-1)}(\mathbb{C}), \quad f \mapsto\left(f, f+f^{\prime}, \ldots, f+f^{(n-1)}\right),
$$

(this modification of the usual jet map ensures that the right hand side is an $n$-tuple of monic polynomials of degree exactly $d$ ). All these maps are related by the following commutative diagram:

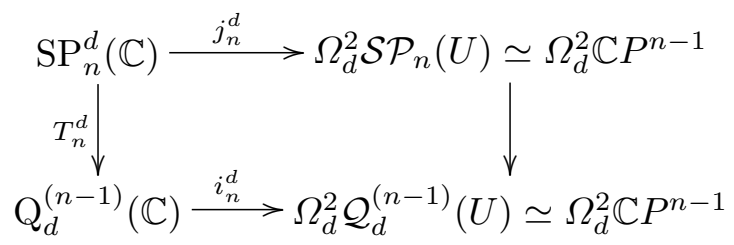

where the right hand vertical map is induced by the (modified) jet map $\mathcal{S P}_{n}(U) \rightarrow \mathcal{Q}^{(n-1)}(U)$. Exactly as in the proof of Theorem 2.4, one can show that this jet map is homotopic to the identity map.

It follows from this that we have a factorization $j_{n}^{d} \simeq i_{n}^{d} \circ T_{n}^{d}$. Hence:

Corollary 2.8. The limit $\left(\right.$ as $d \rightarrow \infty$ ) of $T_{n}^{d}: \mathrm{SP}_{d}^{n}(\mathbb{C}) \rightarrow \mathrm{Q}_{d}^{(n-1)}(\mathbb{C})$ is a homotopy equivalence for $n \geq 3$, and a homology equivalence for $n=2$.

It turns out that there is a much more precise relation between $\mathrm{SP}_{d}^{n}(\mathbb{C})$ and $\mathrm{Q}_{d}^{(n-1)}(\mathbb{C})$. In the introduction we referred to the result proved in $\left[\right.$ GKY2] (a sketch proof was also given in $[\mathrm{Kl}]$ ) that $\mathrm{SP}_{n}^{n d}(\mathbb{C})$ and $\mathrm{Q}_{d}^{(n-1)}(\mathbb{C})$ are homotopy equivalent. (This generalizes an earlier result of Vassiliev ([Va1], [Va2]), which shows that they are stably homotopy equivalent.) To 
provide some motivation for this, we need "unstable" versions of Theorems 2.4 and 2.6. Regarding Theorem 2.4, we have:

TheOREM 2.9. The map $j_{n}^{d}: \mathrm{SP}_{n}^{d}(\mathbb{C}) \rightarrow \Omega_{d}^{2} \mathbb{C} P^{n-1}$ (defined earlier) is a homotopy equivalence up to dimension $(2 n-3)[d / n]$ if $n \geq 3$, and a homology equivalence up to dimension $(2 n-3)[d / n]$ if $n=2$.

Proof. It follows from $[\mathrm{Ar}]$ that the stabilization map $\mathrm{SP}_{n}^{d}(\mathbb{C}) \rightarrow$ $\mathrm{SP}_{n}^{d+1}(\mathbb{C})$ is a homology equivalence up to dimension $(2 n-3)[d / n]$. (Further details concerning this result are given in the Appendix.) Taken together with Theorem 2.4, this gives the homology statement of the theorem. If $n \geq 3$ both spaces are simply connected, so we obtain the homotopy statement as well.

Segal considered the stabilization map $\mathrm{Q}_{d}^{(n-1)}(\mathbb{C}) \rightarrow \mathrm{Q}_{d+1}^{(n-1)}(\mathbb{C})$ in Section 5 of [Se2]. He obtained the following unstable version of Theorem 2.6:

TheOREM 2.10. The inclusion map $i_{n}^{d}: \mathrm{Q}_{d}^{(n-1)}(\mathbb{C}) \rightarrow \Omega_{d}^{2} \mathbb{C} P^{n-1}$ is a homotopy equivalence up to dimension $(2 n-3) d$ if $n \geq 3$, and a homology equivalence up to dimension $(2 n-3) d$ if $n=2$.

The last two theorems show that $\operatorname{SP}_{n}^{n d}(\mathbb{C})$ and $\mathrm{Q}_{d}^{(n-1)}(\mathbb{C})$ have isomorphic homology groups up to dimension $(2 n-3) d-1$, which indicates the plausibility of the result of [GKY2] that they are homotopy equivalent. Analogous results for spaces of the type $\mathrm{P}_{Y, n}^{n d}(X)$ and $\mathrm{Q}_{Y, d}^{n-1}(X)$ (see the next section), where $X$ and $Y$ are $\mathbb{R}$ or $\mathbb{C}$, are proved in [KY1].

\section{Further examples of the same type}

A theorem of Vassiliev. We now turn to Vassiliev's original result (mentioned in the introduction), which concerns real polynomials.

Definition 3.1. $\mathrm{P}_{n}^{d}(\mathbb{R})$ denotes the space of real polynomials of degree $d$ which are monic, and all of whose real roots have multiplicity less than $n$.

The space $\mathrm{P}_{n}^{d}(\mathbb{R})$ is a subspace of $\mathrm{SP}^{d}(\mathbb{C})$ (but it is not a subspace of $\mathrm{SP}_{n}^{d}(\mathbb{C})$ because no conditions are imposed on the nonreal roots). We have a "horizontal scanning map"

$$
\mathrm{P}_{n}^{d}(\mathbb{R}) \rightarrow \Omega \mathrm{P}_{n}(I, \partial I)
$$

where $I=[-1,1]$ and

$\mathrm{P}_{n}(I, \partial I)=\left\{\sum d_{i} x_{i} \in \mathrm{SP}\left(I \times[-1,1], \partial I \times[-1,1] \mid d_{i}<n\right.\right.$ when $\left.x_{i} \in \mathbb{R}\right\}$.

This is defined by associating with a configuration $\alpha$ the map

$$
x \mapsto \alpha \cap \bar{V}(x) \in \mathrm{P}_{n}(\bar{V}(x), \partial \bar{V}(x)) \cong \mathrm{P}_{n}(I, \partial I),
$$

where $V(x)=\{z \in \mathbb{C}|| \operatorname{Re}(z)-x \mid<\varepsilon\}$. 
The analogue of Definition 2.3 here is:

Definition 3.2. (1) For $n \geq 2, \mathcal{P}_{n}^{d}(\mathbb{R})$ denotes the space of (not necessarily monic) real polynomial functions of degree exactly $d$, all of whose real roots have multiplicity less than $n$.

(2) For $n \geq 2$, and any nonempty open subset $X \subseteq \mathbb{R}, \mathcal{P}_{n}^{d}(X)$ denotes the space of real polynomial functions $f(x)=\sum a_{i} x^{i}$ such that every root of $f$ in $X$ has multiplicity less than $n$, and $f$ is not identically zero.

As in the case of complex polynomials, there is a horizontal scanning map

$$
\operatorname{scan}: \mathcal{P}_{n}^{d}(\mathbb{R}) \rightarrow \operatorname{Map}\left(\mathbb{R}, \mathcal{P}_{n}((-1,1))\right), \quad f \mapsto\left(\left.x \mapsto f\right|_{V(x)}\right),
$$

and a jet map

$$
\text { jet }: \mathcal{P}_{n}^{d}(\mathbb{R}) \rightarrow \operatorname{Map}\left(\mathbb{R}, \mathbb{R}^{n}-\{0\}\right),\left.\quad f \mapsto\left(f, f^{\prime}, \ldots, f^{(n-1)}\right)\right|_{\mathbb{R}} .
$$

We have a commutative diagram analogous to the ones in the last section:

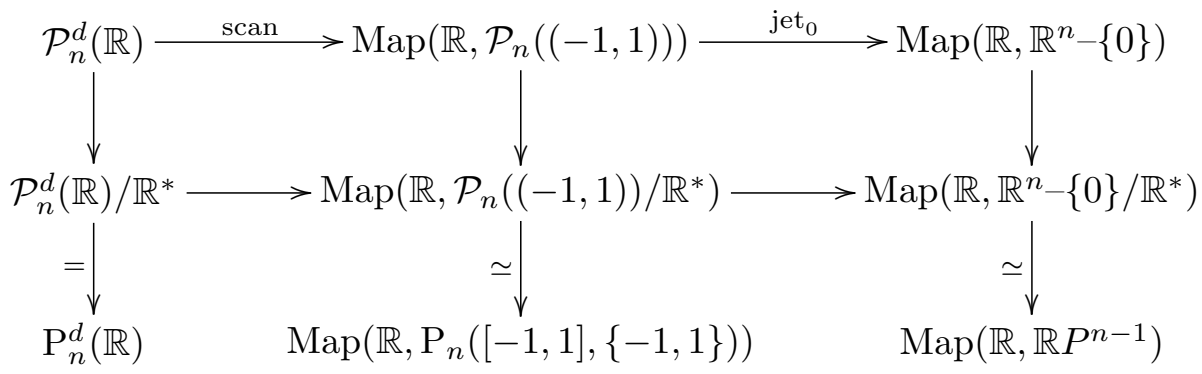

Let $j_{n}^{d}: \mathrm{P}_{n}^{d}(\mathbb{R}) \rightarrow \Omega_{d} \mathbb{R} P^{n-1}$ be the second row of this diagram. We can now give a proof of Vassiliev's result concerning $\mathrm{P}_{n}^{d}(\mathbb{R})$ as $d \rightarrow \infty$.

THEOREM 3.3. For $n \geq 4$,

$$
\lim _{d \rightarrow \infty} j_{n}^{d}: \lim _{d \rightarrow \infty} \mathrm{P}_{n}^{d}(\mathbb{R}) \rightarrow \Omega_{0} \mathbb{R} P^{n-1}
$$

is a homotopy equivalence. For $n=3, \lim _{d \rightarrow \infty} j_{n}^{d}$ is a homology equivalence.

Proof. This is exactly analogous to the proof of Theorem 2.4.

Related examples. There is a family of related examples which includes the spaces $\mathrm{SP}_{n}^{d}(\mathbb{C}), \mathrm{P}_{n}^{d}(\mathbb{R})$ :

Definition 3.4. Let $X, Y$ be subspaces of $\mathbb{C}$. Then $\mathrm{P}_{Y, n}^{d}(X)$ denotes the space of complex monic polynomials $f$ of degree $d$ such that (i) $f(X) \subseteq X$ and (ii) $f$ has no $n$-fold roots in $Y$.

We shall consider the four examples obtained by taking $X, Y=\mathbb{R}$ or $\mathbb{C}$. It will be convenient to express these results in terms of spheres rather than projective spaces; note that $\Omega^{2} S^{2 n-1}$ may be identified with the identity component of $\Omega^{2} \mathbb{C} P^{n-1}$, and that $\Omega S^{2 n-1}$ may be identified with the 
universal covering space of $\Omega \mathbb{C} P^{n-1}$. First, we have $\mathrm{P}_{\mathbb{C}, n}^{d}(\mathbb{C})=\mathrm{SP}_{n}^{d}(\mathbb{C})$ and $\mathrm{P}_{\mathbb{R}, n}^{d}(\mathbb{R})=\mathrm{P}_{n}^{d}(\mathbb{R})$, and we have seen in Theorems 2.4 and 3.3 that these provide finite-dimensional models for the loop spaces $\Omega^{2} \mathbb{C} P^{n-1}$ and $\Omega \mathbb{R} P^{n-1}$.

Concerning $\mathrm{P}_{\mathbb{R}, n}^{d}(\mathbb{C})$ we have:

TheOREM 3.5. For $n \geq 3$, there is a homotopy equivalence

$$
\lim _{d \rightarrow \infty} \mathrm{P}_{\mathbb{R}, n}^{d}(\mathbb{C}) \rightarrow \Omega S^{2 n-1} .
$$

Pr o of. The method of Theorem 3.3 (using a horizontal scanning map) applies equally well here.

Concerning $\mathrm{P}_{\mathbb{C}, n}^{d}(\mathbb{R})$, there is a similar statement, but this time involving the involution $\theta: \mathbb{C} \rightarrow \mathbb{C}, \theta(z)=\bar{z}$ (and its natural extension to $\mathbb{C}^{n}$ ).

ThEOREM 3.6. For $n \geq 3$, there is a homotopy equivalence

$$
\lim _{d \rightarrow \infty} \mathrm{P}_{\mathbb{C}, n}^{d}(\mathbb{R}) \rightarrow\left(\Omega^{2} S^{2 n-1}\right)^{\theta},
$$

where $\left(\Omega^{2} S^{2 n-1}\right)^{\theta}$ is the subspace of $\Omega^{2} S^{2 n-1}$ consisting of maps $f$ which satisfy the condition $\theta(f(z))=f(\theta(z))$ for all $z \in \mathbb{C} \cup\{\infty\}$.

Proof. The method of Theorem 2.4 can be modified by imposing " $\theta$ equivariance" at the appropriate $\left({ }^{3}\right)$ points.

As we pointed out in the introduction, Theorems 2.4, 3.3, 3.5 and 3.6 may be summarized in a single statement. To avoid special cases which occur for low values of $n$, we assume $n \geq 4$ in the following version.

THEOREM 3.7. There is a $\theta$-equivariant homotopy equivalence

$$
\lim _{d \rightarrow \infty} \mathrm{P}_{Y, n}^{d}(\mathbb{C}) \rightarrow \operatorname{Map}\left(Y^{+}, S^{2 n-1}\right)
$$

(if $n \geq 4$ ), where $Y=\mathbb{R}$ or $\mathbb{C}, Y^{+}=\mathbb{R} \cup\{\infty\}$ or $\mathbb{C} \cup\{\infty\}$, and Map indicates the space of basepoint preserving continuous maps.

There is an analogous family of results relating to Segal's $Q_{d}^{(n-1)}(\mathbb{C})$ (the definition of this space was given in $\S 2$ ). We may introduce a space $\mathrm{Q}_{d}^{Y,(n-1)}(X)$ for any subsets $X, Y$ of $\mathbb{C}$, in which the polynomials $p_{1}, \ldots, p_{n}$ are required to satisfy the modified conditions (i) $p_{i}(X) \subseteq X$ and (ii) $p_{1}, \ldots, p_{n}$ have no common root in $Y$. There are four basic examples. First we have $\mathrm{Q}_{d}^{\mathbb{C},(n-1)}(\mathbb{C})=\mathrm{Q}_{d}^{(n-1)}(\mathbb{C})$ and $\mathrm{Q}_{d}^{\mathbb{R},(n-1)}(\mathbb{R})$. Then there are two "mixed" spaces, $Q_{d}^{\mathbb{C},(n-1)}(\mathbb{R})$ and $\mathrm{Q}_{d}^{\mathbb{R},(n-1)}(\mathbb{C})$. The first of these is the space of "real" algebraic maps $\mathbb{C} P^{1} \rightarrow \mathbb{C} P^{n-1}$, and this space (for $n=2$ at least) has already been discussed by Segal in [Se2].

We have the following analogue of Theorem 3.7:

$\left({ }^{3}\right)$ In fact, the validity of the equivariant scanning argument in the case of more general finite group actions has already been noted in [Se3]. 
THEOREM 3.8. There is a $\theta$-equivariant homotopy equivalence

$$
\lim _{d \rightarrow \infty} \mathrm{Q}_{d}^{Y,(n-1)}(\mathbb{C}) \rightarrow \operatorname{Map}\left(Y^{+}, S^{2 n-1}\right)
$$

(if $n \geq 4$ ), where $Y=\mathbb{R}$ or $\mathbb{C}, Y^{+}=\mathbb{R} \cup\{\infty\}$ or $\mathbb{C} \cup\{\infty\}$, and Map indicates the space of basepoint preserving continuous maps.

Spaces of holomorphic maps "with bounded multiplicities". Segal's result on the space $\operatorname{Hol}\left(S^{2}, \mathbb{C} P^{n-1}\right)$ may be generalized by imposing conditions of bounded multiplicity on the various polynomials involved. The appropriate generalization of Definition 2.5 is:

Definition 3.9. For $n, m \geq 2$, let $\mathrm{Q}_{d}^{(n-1), m}(\mathbb{C})$ be the space of $n$-tuples $\left(\alpha_{1}, \ldots, \alpha_{n}\right) \in \mathrm{SP}_{m}^{d}(\mathbb{C}) \times \ldots \times \operatorname{SP}_{m}^{d}(\mathbb{C})$ such that $\alpha_{1} \cap \ldots \cap \alpha_{n}=\emptyset$.

We denote by $\operatorname{Hol}_{d}^{m}\left(S^{2}, \mathbb{C} P^{n-1}\right)$ the subspace of $\operatorname{Hol}_{d}\left(S^{2}, \mathbb{C} P^{n-1}\right)$ which corresponds to $\mathrm{Q}_{d}^{(n-1), m}(\mathbb{C})$. Observe that we have a filtration

$$
\emptyset=\operatorname{Hol}_{d}^{1}\left(S^{2}, \mathbb{C} P^{n-1}\right) \subseteq \ldots \subseteq \operatorname{Hol}_{d}^{d+1}\left(S^{2}, \mathbb{C} P^{n-1}\right)=\operatorname{Hol}_{d}\left(S^{2}, \mathbb{C} P^{n-1}\right)
$$

Corresponding to Definition 2.7 we have:

Definition 3.10. (1) For $n, m \geq 2, \mathcal{Q}_{d}^{(n-1), m}(\mathbb{C})$ denotes the space of $n$ tuples $\left(p_{1}, \ldots, p_{n}\right)$ with $p_{i} \in \mathcal{S P}_{m}^{d}(\mathbb{C})$ such that $p_{1}, \ldots, p_{n}$ have no common root.

(2) For $n, m \geq 2$, and any nonempty open subset $X \subseteq \mathbb{C}, \mathcal{Q}_{d}^{(n-1), m}(X)$ denotes the space of $n$-tuples $\left(p_{1}, \ldots, p_{n}\right)$ with $p_{i} \in \mathcal{S P}_{m}^{d}(X)$ such that $p_{1}, \ldots, p_{n}$ have no common root in $X$.

We can now proceed in the usual way. The only new task is to study the jet map

$$
\begin{gathered}
\operatorname{jet}_{0}: \mathcal{Q}^{(n-1), m}(U) \rightarrow A_{n, m}, \\
\left(p_{1}, \ldots, p_{n}\right) \mapsto\left(\left(p_{1}(0), \ldots, p_{1}^{(m-1)}(0)\right), \ldots,\left(p_{n}(0), \ldots, p_{n}^{(m-1)}(0)\right)\right),
\end{gathered}
$$

where

$$
A_{n, m}=\left\{\left(v_{1}, \ldots, v_{n}\right) \in\left(\mathbb{C}^{m}-\{0\}\right)^{n} \mid\left(\left(v_{1}\right)_{1}, \ldots,\left(v_{n}\right)_{1}\right) \neq(0, \ldots, 0)\right\} .
$$

As in the proof of Theorem 2.4 , it can be shown that jet is a $\left(\mathbb{C}^{*}\right)^{n}$ equivariant homotopy equivalence. This leads to the following generalization of Theorem 2.6, for the analogous map $j_{n, m}^{d}: \mathrm{Q}_{d}^{(n-1), m}(\mathbb{C}) \rightarrow \Omega_{d}^{2} A_{n, m}$ :

Theorem 3.11. Let $n \geq 2$. For $m \geq 3$,

$$
\lim _{d \rightarrow \infty} j_{n, m}^{d}: \lim _{d \rightarrow \infty} \mathrm{Q}_{d}^{(n-1), m}(\mathbb{C}) \rightarrow \Omega_{0}^{2} A_{n, m}
$$

is a homotopy equivalence. For $m=2, \lim _{d \rightarrow \infty} j_{n, m}^{d}$ is a homology equivalence. 
The subset $A_{n, m} /\left(\mathbb{C}^{*}\right)^{n}$ of $\left(\mathbb{C} P^{m-1}\right)^{n}$ consists of elements $\left(\left[v_{1}\right], \ldots,\left[v_{n}\right]\right)$ such that at least one of the points $\left[v_{1}\right], \ldots,\left[v_{n}\right]$ lies in the "big cell" $\{[v] \in$ $\left.\mathbb{C} P^{m-1} \mid(v)_{1} \neq 0\right\} \cong \mathbb{C}^{m-1}$. Hence it is homotopy equivalent to the fat wedge $W_{n}\left(\mathbb{C} P^{m-1}\right)$, i.e. the set of all $\left(\left[v_{1}\right], \ldots,\left[v_{n}\right]\right)$ such that at least one of $\left[v_{1}\right], \ldots,\left[v_{n}\right]$ is equal to the basepoint $[1 ; 0 ; \ldots ; 0]$ of $\mathbb{C} P^{m-1}$. Thus, Theorem 3.11 gives a homotopy equivalence

$$
\lim _{d \rightarrow \infty} \mathrm{Q}_{d}^{(n-1), m}(\mathbb{C}) \rightarrow \Omega_{0}^{2} W_{n}\left(\mathbb{C} P^{m-1}\right) .
$$

This shows that the space $A_{n, m}$ has a natural interpretation, which is consistent with Segal's original approach (for the case $m=\infty$ ).

\section{The h-Principle}

Spaces of polynomials with roots of bounded multiplicity. As preparation for the h-Principle, we shall give a general formulation which includes all the examples considered so far in this paper. Let $\mathcal{F}_{D}(\mathbb{C})$ denote the space of $n$-tuples $\left(p_{1}, \ldots, p_{n}\right)$ of (not identically zero) complex polynomials satisfying conditions of the following types:

(i) "degree conditions" (e.g. $\operatorname{deg} p_{i}=d_{i}$, where $D=\left(d_{1}, \ldots, d_{n}\right)$ is fixed),

(ii) "coprime conditions" (e.g. certain subsets $p_{i_{1}}, \ldots, p_{i_{k}}$ of $p_{1}, \ldots, p_{n}$ have no common factor),

(iii) "bounded multiplicity conditions" (e.g. all roots of $p_{i}$ have multiplicity less than $m_{i}$, where $M=\left(m_{1}, \ldots, m_{n}\right)$ is fixed).

Concrete examples of spaces $\mathcal{F}_{D}(\mathbb{C})$ satisfying conditions of type (i) and (ii) are provided $\left({ }^{4}\right)$ by the spaces $\operatorname{Hol}_{D}\left(S^{2}, X_{\Delta}\right)$, where $X_{\Delta}$ is a smooth toric variety defined by a fan $\Delta$ (see [GKY1], [Gu2]). These examples may be modified in obvious ways by imposing conditions of type (iii). We may also consider the analogues of the earlier spaces $\mathrm{P}_{Y, n}^{d}(X)$ or $\mathrm{Q}_{d}^{Y,(n-1)}(X)$ for $\mathcal{F}_{D}(\mathbb{C})$.

We regard $\left(p_{1}, \ldots, p_{n}\right)$ as a holomorphic map $\mathbb{C} \rightarrow \mathbb{C}^{n}-A$, where $A$ is the union of linear subspaces corresponding to condition (ii). In the case of $\operatorname{Hol}_{D}\left(S^{2}, X_{\Delta}\right)$, the arrangement $A$ depends only on the fan of $X$, so we write $A=A_{\Delta}$. We then have $X_{\Delta} \cong \mathbb{C}^{n}-A_{\Delta} /\left(\mathbb{C}^{*}\right)^{n-\operatorname{dim} X_{\Delta}}$ for a certain (free) action of $\left(\mathbb{C}^{*}\right)^{n-\operatorname{dim} X_{\Delta}}$ (see [Co] and [Gu2]).

For a nonempty subset $X \subseteq \mathbb{C}$, we define $\mathcal{F}(X)$ in the same way, except that conditions (ii) and (iii) now apply only to the roots of $p_{1}, \ldots, p_{n}$ which lie in $X$ (and condition (i) is omitted). Just as in $\S 2$ and $\S 3$, we have a jet map

$\left({ }^{4}\right)$ More precisely, the space $\mathcal{F}_{D}(\mathbb{C}) /\left(\mathbb{C}^{*}\right)^{n}$ of $n$-tuples of monic polynomials corresponds to the space of based holomorphic maps. 


$$
\text { jet : } \mathcal{F}_{D}(\mathbb{C}) \rightarrow \operatorname{Map}\left(\mathbb{C}, \mathbb{C}^{n^{\prime}}-A^{\prime}\right),
$$

where $\mathbb{C}^{n^{\prime}}-A^{\prime}$ is the "prolongation" of $\mathbb{C}^{n}-A$ determined by condition (iii). This factors as the top row of the following diagram:

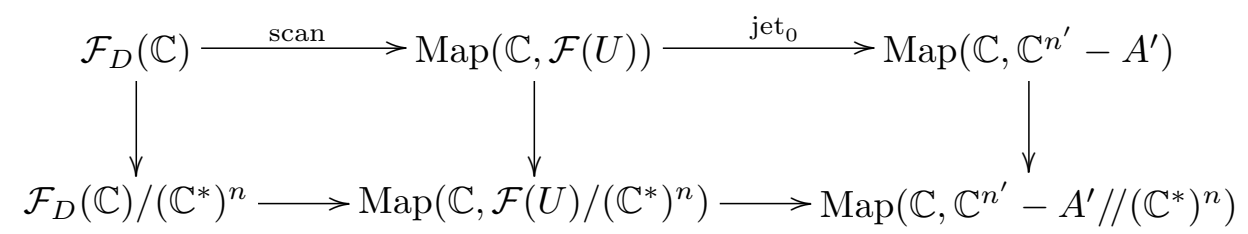

The action of $\left(\mathbb{C}^{*}\right)^{n}$ on $\mathcal{F}(U)$ is always free. If the action of $\left(\mathbb{C}^{*}\right)^{n}$ on $\mathbb{C}^{n^{\prime}}-$ $A^{\prime}$ is free, we may replace the homotopy quotient $\mathbb{C}^{n^{\prime}}-A^{\prime} / /\left(\mathbb{C}^{*}\right)^{n}$ by the ordinary quotient.

As in the proof of Theorem 2.4, we can show:

Lemma 4.1. The map jet $_{0}: \mathcal{F}(U) \rightarrow \mathbb{C}^{n^{\prime}}-A^{\prime}$ is a $\left(\mathbb{C}^{*}\right)^{n}$-equivariant homotopy equivalence.

Because of the interpretation of $\mathcal{F}(\mathbb{C}) /\left(\mathbb{C}^{*}\right)^{n}$ and $\mathcal{F}(U) /\left(\mathbb{C}^{*}\right)^{n}$ as configuration spaces, the scanning method (as in Theorem 2.2) leads to:

Lemma 4.2. The map $\operatorname{scan} /\left(\mathbb{C}^{*}\right)^{n}: \mathcal{F}_{D}(\mathbb{C}) /\left(\mathbb{C}^{*}\right)^{n} \rightarrow \Omega_{D}^{2} \mathcal{F}(U) /\left(\mathbb{C}^{*}\right)^{n}$ is a homotopy equivalence in the limit $D \rightarrow \infty$.

We deduce (from the lemmas and the diagram) the following generalization of Theorems 2.2 and 2.6:

TheOREM 4.3. The jet map induces a map

$$
j_{D}: \mathcal{F}_{D}(\mathbb{C}) /\left(\mathbb{C}^{*}\right)^{n} \rightarrow \Omega_{D}^{2}\left(\mathbb{C}^{n^{\prime}}-A^{\prime}\right),
$$

and $\lim _{D \rightarrow \infty} j_{D}$ is a homotopy equivalence (or homology equivalence, if the roots of any polynomial in the definition of $\mathcal{F}_{D}(\mathbb{C})$ are required by condition (iii) to be distinct).

In the special case where $\mathcal{F}_{D}(\mathbb{C}) /\left(\mathbb{C}^{*}\right)^{n}=\operatorname{Hol}_{D}\left(S^{2}, X_{\Delta}\right)$ for some toric variety $X_{\Delta}$, we have $\mathbb{C}^{n^{\prime}}-A^{\prime}=\mathbb{C}^{n}-A$ and $\Omega_{D}^{2}\left(\mathbb{C}^{n^{\prime}}-A^{\prime}\right) \simeq \Omega_{D}^{2}\left(\mathbb{C}^{n}-A\right) \simeq$ $\Omega_{D}^{2} X_{\Delta}$. In this case the map $j_{D}$ of Theorem 4.3 may be identified with the natural inclusion $\operatorname{Hol}_{D}\left(S^{2}, X_{\Delta}\right) \rightarrow \Omega_{D}^{2} X_{\Delta}$.

In general, Theorem 4.3 exhibits $\mathcal{F}_{D}(\mathbb{C}) /\left(\mathbb{C}^{*}\right)^{n}$ (which is a "space of polynomials with roots of bounded multiplicity") as a model for the double loop space of $\mathbb{C}^{n^{\prime}}-A^{\prime}$. This, in turn, is homotopy equivalent to the double loop space of a "generalized wedge product" of finite-dimensional complex projective spaces (see [GKY1] and the remarks following Theorem 3.11 in the previous section).

The h-Principle. Let $M, N$ be smooth manifolds. A smooth map $f$ : $M \rightarrow N$ may be regarded as a section of the trivial bundle $M \times N \rightarrow M$, 
and its $k$-jet $j_{k}(f): M \rightarrow J_{k}(M, N)$ is then a section of the $k$-jet bundle $J_{k}(M, N) \rightarrow M$. Thus we have a map

$$
j_{k}: \operatorname{Map}(M, N) \rightarrow \operatorname{Sec}\left(J_{k}(M, N)\right), \quad f \mapsto j_{k}(f),
$$

where Map and Sec denote smooth maps and smooth sections, respectively. This map is not in general surjective; an element $s$ of $\operatorname{Sec}\left(J_{k}(M, N)\right)$ is said to be holonomic or integrable if there exists an element $f$ of $\operatorname{Map}(M, N)$ such that $j_{k}(f)=s$.

More generally, if $S$ is a closed subspace of $J_{k}(M, N)$, we define

$$
\begin{aligned}
\operatorname{Map}^{S}(M, N) & =\left\{f \in \operatorname{Map}(M, N) \mid j_{k}(f)(M) \cap S=\emptyset\right\}, \\
\operatorname{Sec}^{S}\left(J_{k}(M, N)\right) & =\left\{s \in \operatorname{Sec}\left(J_{k}(M, N)\right) \mid s(M) \cap S=\emptyset\right\} .
\end{aligned}
$$

Then we have

$$
j_{k}^{S}: \operatorname{Map}^{S}(M, N) \rightarrow \operatorname{Sec}^{S}\left(J_{k}(M, N)\right), \quad f \mapsto j_{k}(f),
$$

and the image of this map consists of all sections which satisfy the integrability condition.

The Smale-Hirsch Principle, or the (parametrized) h-Principle of Gromov, says that, under certain conditions on $M, N$ and $S$, the $\operatorname{map} j_{k}^{S}$ is a homotopy equivalence. Under such favourable conditions, the integrability condition is therefore "irrelevant from the point of view of topology". An extensive treatment of the h-Principle and its generalizations can be found in $[\mathrm{Gr}]$. Even to summarize this work briefly here would not be feasible; we just mention that examples of situations where the Principle holds are often found when $M$ and $N$ are open manifolds, or when $S$ is not too large.

For example, let $\operatorname{dim} M<\operatorname{dim} N$, and let $J_{1}(M, N)-S$ be defined by the condition that the derivative has maximal rank. Thus, $\operatorname{Map}^{S}(M, N)$ is the space of smooth immersions of $M$ in $N$. Smale and Hirsch studied immersions of $M=S^{m}$ in $N=\mathbb{R}^{n}$ and discovered that regular homotopy classes of such immersions are in one-to-one correspondence with the elements of $\pi_{m} V_{m}\left(\mathbb{R}^{n}\right)$, where $V_{m}\left(\mathbb{R}^{n}\right)$ is the Stiefel manifold of $m$-frames in $\mathbb{R}^{n}$. This is consistent with the h-Principle, as $\operatorname{Sec}^{S}\left(J_{1}(M, N)\right)$ is homotopy equivalent to $\operatorname{Map}\left(S^{m}, V_{m}\left(\mathbb{R}^{n}\right)\right)$ in this case.

As another example, let $M$ and $N$ be complex manifolds, and take $J_{1}(M, N)-S$ to be defined by the condition that the derivative is $\mathbb{C}$-linear. In this case we have $\operatorname{Map}^{S}(M, N)=\operatorname{Hol}(M, N)$. On the other hand the space $\operatorname{Sec}^{S}\left(J_{1}(M, N)\right)$ is homotopy equivalent to $\operatorname{Map}(M, N)$ (since the space of $\mathbb{C}$-linear transformations is an $\mathbb{R}$-linear subspace of the space of $\mathbb{R}$-linear transformations). So the h-Principle holds if and only if the inclusion $\operatorname{Hol}(M, N) \rightarrow \operatorname{Map}(M, N)$ is a homotopy equivalence. This is certainly false in general (see $[\mathrm{Gr}]$ ), in particular when $M=N=S^{2}$. However, Segal's theorem indicates that something can be salvaged in this case. In 
the remainder of this section we shall describe how Theorem 4.3 may be approached via the h-Principle.

Our starting point is Vassiliev's observation that $\lim _{D \rightarrow \infty} \mathcal{F}_{D}(\mathbb{C})$ is (weakly) homotopy equivalent to the space of smooth maps $f: \mathbb{C} \rightarrow \mathbb{C}^{n}-A$ such that the image of jet $(f)$ lies in $\mathbb{C}^{n^{\prime}}-A^{\prime}$ and such that jet $(f)$ satisfies the same "condition at $\infty$ " as elements of $\mathcal{F}_{D}(\mathbb{C})$. Let us denote this space by $\mathrm{F}^{*}(\mathbb{C})$. Theorem 4.3 is therefore equivalent to the statement that the jet map

$$
j: \mathrm{F}^{*}(\mathbb{C}) /\left(\mathbb{C}^{*}\right)^{n} \rightarrow \Omega_{0}^{2}\left(\mathbb{C}^{n^{\prime}}-A^{\prime}\right)
$$

is a homotopy equivalence. This statement, which concerns only smooth maps, may be deduced from the h-Principle, as we shall now explain.

Let us denote by $F(\mathbb{C}$ ) (and similarly for $F(X)$ ) the space of smooth maps $f: \mathbb{C} \rightarrow \mathbb{C}^{n}-A$ such that the image of jet $(f)$ lies in $\mathbb{C}^{n^{\prime}}-A^{\prime}$. We claim that the $h$-Principle is valid in this case, at least for any surface $X$ which is constructed by successively attaching two-dimensional disks to the two-dimensional disk $U$. This may be proved by induction. For the case $X=U$, the validity of the h-Principle is proved by "shrinking $U$ down to a point". The inductive step is achieved by using the fact that the functor $F$ converts (certain) cofibrations into fibrations (see the last part of our explanation of the proof of Theorem 2.2, for a special case). The details of this argument are explained, in greater generality, in [Ha] and [Po].

Now we consider the usual commutative diagram, but this time for the functor $F$ (rather than for algebraic maps):

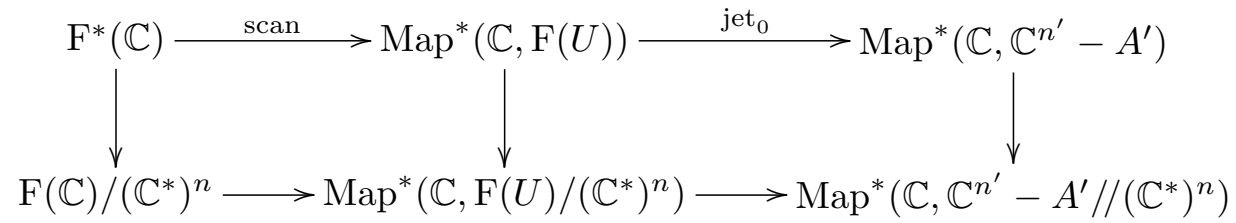

The map $j$ is given by the bottom row. It suffices, therefore, to show that the top row is a homotopy equivalence. (The conditions at $\infty$ in the top row are defined to be those which descend to the given conditions at $\infty$ in the bottom row; the symbol Map* indicates that these conditions are in force.) The fact that the top row is a homotopy equivalence requires two observations:

(1) the jet map $\mathrm{F}(U) \rightarrow \mathbb{C}^{n^{\prime}}-A^{\prime}$ is a homotopy equivalence, and

$(2)$ the scanning map $\mathrm{F}^{*}(\mathbb{C}) \rightarrow \operatorname{Map}^{*}(\mathbb{C}, \mathrm{F}(U))$ is a homotopy equivalence.

We have already seen that (1) is true, as it begins the inductive argument for the proof of the h-Principle for F. To prove (2), we consider the following commutative diagram: 


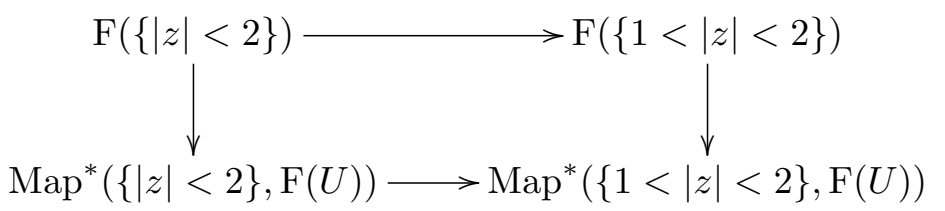

in which the horizontal maps are given by restriction and the vertical maps are given by scanning. The horizontal maps are fibrations, and-by the hPrinciple for the cases where $X$ is a disk or an annulus - the vertical maps are homotopy equivalences. Hence the map of fibres is also a homotopy equivalence. But this is the scanning map $\mathrm{F}^{*}(U) \rightarrow \operatorname{Map}^{*}(U, \mathrm{~F}(U))$, which is homotopic to the map of (2).

Appendix. For completeness, we shall give here the proof of Arnold's result on the homology of $\mathrm{SP}_{n}^{d}(\mathbb{C})$, which was used in the proof of Theorem 2.9. Rather than quote directly from $[\mathrm{Ar}]$ (where a minor error occurs in the statement), we shall sketch an argument along the lines of [Va1].

Theorem A1 ([Ar]). For $n, d \geq 2$, the stabilization map $\mathrm{SP}_{n}^{d}(\mathbb{C}) \rightarrow$ $\mathrm{SP}_{n}^{d+1}(\mathbb{C})$ is a homology equivalence up to dimension $N(d, n)$, where

$$
N(d, n)= \begin{cases}(2 n-3)[d / n] & \text { if }[d / n]<[(d+1) / n], \\ \infty & \text { if }[d / n]=[(d+1) / n] .\end{cases}
$$

Proof. For brevity we shall write $\mathrm{SP}_{n}^{d}=\mathrm{SP}_{n}^{d}(\mathbb{C})$ and $\mathrm{SP}^{d}=\mathrm{SP}^{d}(\mathbb{C})$, and we omit explicit mention of coefficients in (co)homology. Let $\Sigma_{n}^{d} \subseteq \mathrm{SP}^{d}$ denote the discriminant variety consisting of all polynomials $f \in \mathrm{SP}^{d}$ which have at least one root of multiplicity $n$. Since $\mathrm{SP}_{n}^{d}=\mathrm{SP}^{d}-\Sigma_{n}^{d}$, Alexander duality gives

$$
H^{k}\left(\mathrm{SP}_{n}^{d}\right) \cong \bar{H}_{2 d-k-1}\left(\Sigma_{n}^{d}\right) \quad \text { if } 0<k<2 d,
$$

where we use the notation $\bar{H}_{*}(X)=H_{*}(\bar{X})$, with $\bar{X}=X \cup\{\infty\}$ the onepoint compactification of (a locally compact space) $X$.

Let $I: \mathbb{C} \rightarrow \mathbb{C}^{[d / n]}$ be the Veronese embedding, $I(z)=\left(z, z^{2}, \ldots, z^{[d / n]}\right)$. Let $f \in \Sigma_{n}^{d}$. Assume that $f$ has at least $s$ distinct roots $z_{1}, \ldots, z_{s}$ of multiplicity $n$. In this case, we denote by $\Delta\left(f ;\left\{z_{1}, \ldots, z_{s}\right\}\right)$ the $(s-1)$ dimensional open simplex in $\mathbb{C}^{[d / n]}$ with vertices $I\left(z_{1}\right), \ldots, I\left(z_{s}\right)$. (Note that since $s \leq[d / n]$, the points $I\left(z_{1}\right), \ldots, I\left(z_{s}\right)$ are in general position.)

Define the geometric resolution $G=G\left(\Sigma_{n}^{d}\right)$ of $\Sigma_{n}^{d}$ by

$$
G=G\left(\Sigma_{n}^{d}\right)=\bigcup_{f \in \Sigma_{n}^{d},\left\{z_{1}, \ldots, z_{s}\right\}}\{f\} \times \Delta\left(f ;\left\{z_{1}, \ldots, z_{s}\right\}\right) \subseteq \Sigma_{n}^{d} \times \mathbb{C}^{[d / n]} .
$$

Projection onto the first factor is a surjective open proper map $G \rightarrow \Sigma_{n}^{d}$, and this extends naturally to a map $\pi: \bar{G} \rightarrow \overline{\Sigma_{n}^{d}}$. It is known that $\pi$ is a homotopy equivalence ([Va1]). 
Define the subspaces $\left\{F_{p}\right\}_{p \geq 0}$ of $\bar{G}$ by

$$
F_{p}=\{\infty\} \cup\left(\bigcup_{f \in \Sigma_{n}^{d}, s \leq p}\{f\} \times \Delta\left(f ;\left\{z_{1}, \ldots, z_{s}\right\}\right) .\right.
$$

There is an increasing filtration

$$
F_{0} \subseteq F_{1} \subseteq \ldots \subseteq F_{[d / n]}=F_{[d / n]+1}=\ldots=\bar{G} \simeq \overline{\Sigma_{n}^{d}}
$$

and so we have a homology spectral sequence $E_{p, q}^{1}=\bar{H}_{p+q}\left(F_{p}-F_{p-1}\right) \Rightarrow$ $\bar{H}_{p+q}\left(\Sigma_{n}^{d}\right)$.

If we take $E_{p, q}^{r}=E_{r}^{p, 2 d-1-q}$, we obtain from (*) a cohomology spectral sequence

$$
\mathcal{F}_{n}^{d}=\left\{E_{r}^{p, q}, d_{r}: E_{r}^{p, q} \rightarrow E_{r}^{p-r, q+1-r}\right\}, \quad E_{r}^{p, q} \Rightarrow H^{q-p}\left(\mathrm{SP}_{n}^{d}\right) .
$$

Since there is a fibre bundle $F_{p}-F_{p-1} \rightarrow C_{p}(\mathbb{C})$ with fibre homeomorphic to $\mathbb{R}^{2 d-1-(2 n-1) p}$ if $1 \leq p \leq[d / n]$, it follows from the Thom isomorphism theorem and Poincaré duality that

$$
E_{1}^{p, q}= \begin{cases}H^{(2-2 n) p+q}\left(C_{p}(\mathbb{C})\right) & \text { if } 1 \leq p \leq[d / n], \\ 0 & \text { otherwise. }\end{cases}
$$

Similarly we have a cohomology spectral sequence

$$
\mathcal{F}_{n}^{d+1}=\left\{{ }^{\prime} E_{r}^{p, q}, d_{r}^{\prime}:{ }^{\prime} E_{r}^{p, q} \rightarrow{ }^{\prime} E_{r}^{p-r, q+1-r}\right\}, \quad{ }^{\prime} E_{r}^{p, q} \Rightarrow H^{q-p}\left(\mathrm{SP}_{n}^{d+1}\right),
$$

such that

$$
{ }^{\prime} E_{1}^{p, q}= \begin{cases}H^{(2-2 n) p+q}\left(C_{p}(\mathbb{C})\right) & \text { if } 1 \leq p \leq[(d+1) / n], \\ 0 & \text { otherwise }\end{cases}
$$

Note that the stabilization map $\mathrm{SP}_{n}^{d} \rightarrow \mathrm{SP}_{n}^{d+1}$ extends naturally to a map SP${ }^{d} \rightarrow \mathrm{SP}^{d+1}$ and this induces a map $\Sigma_{n}^{d} \rightarrow \Sigma_{n}^{d+1}$. This map extends to the open embedding $\Sigma_{n}^{d} \times \mathbb{C} \rightarrow \Sigma_{n}^{d+1}$ (up to homotopy), which preserves the corresponding filtrations. Because one-point compactification is contravariant for open embeddings, we obtain a map $\bar{s}: \bar{\Sigma}_{n}^{d+1} \rightarrow \overline{\Sigma_{n}^{d} \times \mathbb{C}}=\bar{\Sigma}_{n}^{d} \wedge S^{2}$. Hence the stabilization map $H^{j}\left(\mathrm{SP}_{n}^{d+1}\right) \rightarrow H^{j}\left(\mathrm{SP}_{n}^{d}\right)$ corresponds to the map

$$
\bar{H}_{2 d+1-j}\left(\Sigma_{n}^{d+1}\right) \rightarrow \bar{H}_{2 d-1-j}\left(\Sigma_{n}^{d}\right)
$$

which is the composition

$$
\bar{H}_{2 d+1-j}\left(\Sigma_{n}^{d+1}\right) \stackrel{\bar{s}_{*}}{\longrightarrow} \bar{H}_{2 d+1-j}\left(\Sigma_{n}^{d} \times \mathbb{C}\right) \stackrel{\text { suspension }}{\cong} \bar{H}_{2 d-1-j}\left(\Sigma_{n}^{d}\right) .
$$

Since the above homomorphism preserves the filtrations, this induces a homomorphism of spectral sequences $\left\{\phi_{r}^{p, q}:{ }^{\prime} E_{r}^{p, q} \rightarrow E_{r}^{p, q} \mid r \geq 1,(p, q) \in\right.$ $\mathbb{Z} \times \mathbb{Z}\}$ 
As the corresponding maps between filtrations are natural, the diagram

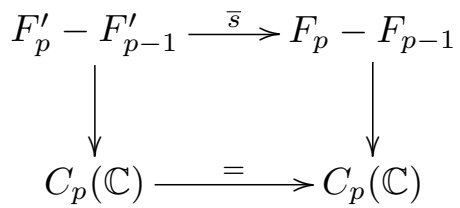

is commutative (for $1 \leq p \leq[d / n]$ ). From the above description of $F_{p}-F_{p-1}$ as a bundle, we have:

(i) If $[d / n]=[(d+1) / n]$, then $\phi_{1}^{p, q}$ is an isomorphism for all $p, q$.

(ii) If $[d / n]<[(d+1) / n]$, then $\phi_{1}^{p, q}$ is an isomorphism if $p \leq 0$, and if $1 \leq p \leq[d / n]$ and $(2-2 n) p+q \geq 0$.

We now apply the comparison theorem for spectral sequences. For (i), the result is immediate, so assume that $[d / n]<[(d+1) / n]$. Then since

$$
(2-2 n) p+q \geq 0 \text { if and only if } q \geq(2 n-2) p,
$$

for $1 \leq p \leq[d / n]$ the dimension $q-p \geq(2 n-3) p$ attains the maximal value $(2 n-3)[d / n]$. Hence the induced homomorphism $H^{j}\left(\mathrm{SP}^{d+1}\right) \rightarrow H^{j}\left(\mathrm{SP}_{n}^{d}\right)$ is an isomorphism if $j \leq(2 n-3)[d / n]$. By the universal coefficients theorem, the same result is valid for homology groups, so the proof is complete.

Corollary A2 $([\mathrm{Ar}])$. If $[d / n]=[(d+1) / n]$ and $n \geq 3$, the stabilization map $\mathrm{SP}_{n}^{d}(\mathbb{C}) \rightarrow \mathrm{SP}_{n}^{d+1}(\mathbb{C})$ is a homotopy equivalence.

\section{References}

[Ar] V. I. Arnold, Some topological invariants of algebraic functions, Trans. Moscow Math. Soc. 21 (1970), 30-52.

[Bo] C.-F. Bödigheimer, Stable splittings of mapping spaces, in: Algebraic Topology, H. R. Miller and D. C. Ravenel (eds.), Lecture Notes in Math. 1286, Springer, 1987, 174-187.

[CCMM] F. R. Cohen, R. L. Cohen, B. M. Mann and R. J. Milgram, The topology of rational functions and divisors of surfaces, Acta Math. 166 (1991), 163-221.

[CJS] R. L. Cohen, J. D. S. Jones and G. B. Segal, Floer's infinite dimensional Morse theory and homotopy theory, in: The Floer Memorial Volume, H. Hofer, C. H. Taubes, A. Weinstein and E. Zehnder (eds.), Progr. Math. 133, Birkhäuser, 1995, 297-325.

[Co] D. A. Cox, The homogeneous coordinate ring of a toric variety, J. Algebraic Geom. 4 (1995), 17-50.

[Fu] K. Fukaya, Topological field theory and Morse theory, Sugaku Expositions 10 (1997), 19-39 (translation from Sūgaku 46 (1994), 289-307).

[GM] M. Goresky and R. MacPherson, Stratified Morse Theory, Springer, 1988.

[Gr] M. Gromov, Partial Differential Relations, Springer, 1986.

[Gu1] M. A. Guest, On the space of holomorphic maps from the Riemann sphere to the quadric cone, Quart. J. Math. 45 (1994), 57-75. 
[Gu2] M. A. Guest, The topology of the space of rational curves on a toric variety, Acta Math. 174 (1995), 119-145.

[GKY1] M. A. Guest, A. Kozlowski and K. Yamaguchi, The topology of spaces of coprime polynomials, Math. Z. 217 (1994), 435-446.

[GKY2] - - - - Stable splitting of the space of polynomials with roots of bounded multiplicity, J. Math. Kyoto Univ. 38 (1998), 351-366.

[Ha] A. Haefliger, Lectures on the theorem of Gromov, in: Proc. of Liverpool Singularities Symposium II, Lecture Notes in Math. 209, C. T. C. Wall (ed.), Springer, 1970, 128-141.

[JS] I. M. James and G. B. Segal, On equivariant homotopy type, Topology 17 (1978), 267-272.

[Kl1] S. Kallel, Particle spaces on manifolds and generalized Poincaré dualities, preprint.

[Kl2] - The topology of spaces of maps from a Riemann surface into complex projective space, preprint.

[Kt] F. Kato, Master's Thesis, Shinshu University, 1994 (in Japanese).

[KY] A. Kozlowski and K. Yamaguchi, Topology of complements of discriminants and resultants, preprint.

[Mc1] D. McDuff, Configuration spaces of positive and negative particles, Topology 14 (1975), 91-107.

[Mc2] -, Configuration spaces, in: K-Theory and Operator Algebras, Lecture Notes in Math. 575, Springer, 1977, 88-95.

[Po] V. Poenaru, Homotopy theory and differential singularities, in: Manifolds (Amsterdam, 1970), Lecture Notes in Math. 197, N. H. Kuiper (ed.), Springer, 1970, 106-133.

[Se1] G. B. Segal, Configuration spaces and iterated loop spaces, Invent. Math. 21 (1973), 213-221.

[Se2] -, The topology of spaces of rational functions, Acta Math. 143 (1979), 39-72.

[Se3] - Some results in equivariant homotopy theory, unpublished manuscript.

[Va1] V. A. Vassiliev, Complements of Discriminants of Smooth Maps: Topology and Applications, Transl. Math. Monographs 98, Amer. Math. Soc., 1992 (rev. ed. 1994).

[Va2] - , Topology of discriminants and their complements, in: Proc. Internat. Congress Math. 1994, Birkhäuser, 209-226.

Department of Mathematics

University of Rochester

Rochester, NY 14627, U.S.A.

E-mail: martin@math.rochester.edu

and

Department of Mathematics

Tokyo Metropolitan University

Minami-Ohsawa 1-1, Hachioji-shi

Tokyo 192-0397, Japan

E-mail:martin@comp.metro-u.ac.jp
Department of Mathematics Toyama International University Kaminikawa, Toyama 930-1262, Japan E-mail: andrzej@tuins.ac.jp

Department of Mathematics The University of Electro-Communications Chofu, Tokyo 182-8585, Japan E-mail: kohhei@prime.e-one.uec.ac.jp 